\title{
Lung Function Classification of Smartphone Recordings Comparison of Signal Processing and Machine Learning Combination Sets
}

\author{
João Teixeira ${ }^{1}$, Luís Teixeira ${ }^{2}$, João Fonseca ${ }^{3}$ and Tiago Jacinto ${ }^{3}$ \\ ${ }^{1}$ Department of Electrical and Computer Engineering, University of Porto, Porto, Portugal \\ ${ }^{2}$ Department of Informatics Engineering, University of Porto, Porto, Portugal \\ ${ }^{3}$ Department of Health Information and Decision Sciences, University of Porto, Porto, Portugal
}

Keywords: Asthma, Breath, COPD, Machine Learning, Signal Processing, Smartphone, Spirometry.

\begin{abstract}
Worldwide, over 250 million people are affected by chronic lung conditions such as Asthma and COPD. These can cause breathlessness, a harsh decrease in quality of life and, if not detected and duly managed, even death. In this paper, we aim to find the best and most efficient combination of signal processing and machine learning approaches to produce a smartphone application that could accurately classify lung function, using microphone recordings as the only input. A total of 61 patients performed the forced expiration maneuver providing a dataset of 101 recordings. The signal processing comparison experiments were conducted in a backward selection approach, reducing from 54 to 12 final envelopes, per recording. The classification experiments focused first on differentiating Normal from Abnormal lung function, and second in multiple lung function patterns. The results from this project encourage further development of the system.
\end{abstract}

\section{INTRODUCTION}

Chronic respiratory diseases such as Asthma and Chronic Obstructive Pulmonary Disease (COPD) are incurable, yet treatable and their early detection is crucial to provide a better quality of life. Major risk factors include air pollution, tobacco smoking and occupational environments containing dust and chemicals. The World Health Organization (WHO) estimates that over 250 million people suffer from asthma and COPD (World Health Organization, 2013a) and more than 3 million people died of COPD in 2005 (World Health Organization, 2013b).

Spirometry is the measurement of breath, i.e., is the most popular noninvasive set of timed tests that enables to measure the mechanical properties of the lungs, also named pulmonary function (Pierce, 2005). The keystone test is the Forced Expiratory Maneuver (FEM) where the patient fully inspires and then forcefully exhales all the air available, as fast as possible.

The increasing use of smartphones has enabled the emergence of several health related systems. Their computational power is ever increasing and, equipped with multiple sensors, it is possible to develop disease prevention, diagnosis and monitoring applications.

The aim of this paper is to compare several groups of methods and clinical parameters in order to find the most relevant, most efficient and faster combination to produce a smartphone app for measuring and classifying lung function. The system's input is restricted to the smartphone's built-in microphone, in order to avoid external components.

\section{BACKGROUND AND RELATED WORK}

Traditional spirometers accurately measure a wide range of lung function parameters but have the disadvantage of being very expensive and being of sizable dimensions.

Portable spirometers were developed to meet the needs of home spirometry and thus, they disregard many unused functions and measurements. Additionally, their cost and dimensions are smaller.

The most recent spirometers use laptops as computational platforms and airflow sensors that use USB connections. This enabled to reuse the computing platform and easily transport the system.

The next logical step concerning portability and affordability involves lung function estimation with smartphones' microphones. Some studies have already been conducted in order to accurately mea- 
sure the clinical parameters (Larson et al., 2012), (Xu et al., 2013), and also considering robustness to ambient noise (van Stein, 2013).

\section{DATA COLLECTION PROCEDURE}

The dataset is composed by 101 recordings from 61 caucasian adult patients performing the forced expiration maneuver, without any mouthpiece, at an arm's length. Some recordings were gathered on a controlled environment with low background noise, however more than $80 \%$ of the recordings experienced background noise such as physicians giving verbal incentive, talking voices and small machine noises at a short distance. The recordings were made using a Samsung GT-I9000.

Each recording is accompanied by the patient's anthropometric parameters (age, height, weight and gender), clinical parameters, and classification of the patients lung function provided by the recording physician. The clinical parameters were obtained by performing the FEM to one of the spirometers available (MIR SpiroDoc, Carefusion Jaeger IOS). The classification types are normal and abnormal (obstruction, restriction or mixed).

The included patients were part of the clinical study Control and Burden of Asthma and Rhinitis (ICAR), patients attending the allergology clinic from CUF Porto Institute (ICP) or from CUF Porto Hospital (HCP). Data collection occurred between April 3rd and June 5th 2014.

\section{ALGORITHMS AND SYSTEM ARCHITECTURE}

\subsection{Signal Processing}

The system's input consists of microphone recordings which are AC coupled, uncalibrated signals that represent air pressure. The signal processing pipeline can be divided in four portions: automatic signal segmentation, signal pre-processing, envelope generation and envelope processing. Figure 1 shows the initial architecture for the signal processing part without the signal segmentation.

\subsubsection{Automatic Signal Segmentation}

The audio input was initially segmented in order to remove non expiration sounds, such as the inspiration

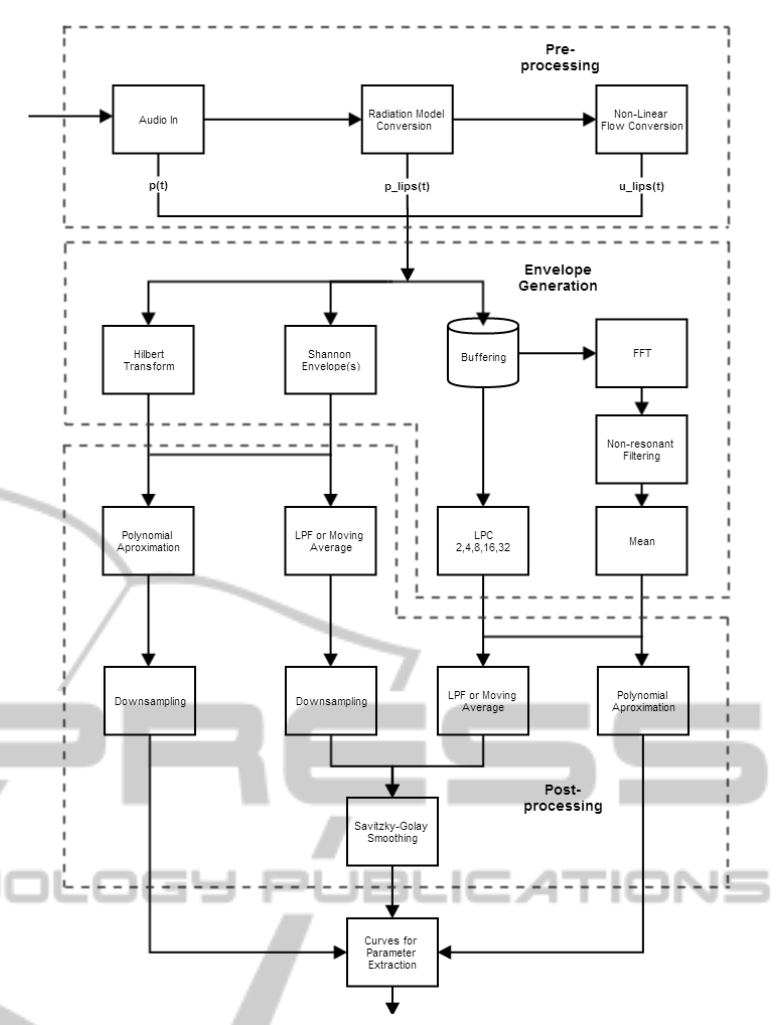

Figure 1: Initial Signal Processing System.

portion of the maneuver and ambient noise. The definition and cropping the beginning of the sound was accomplished using a modified version of the BackExtrapolation algorithm (Miller et al., 2005).

First, an LPC envelope of the signal is obtained, as it will be described further on the paper, then the minimum value between inspiration and expiration peaks is found and the initial part is removed. Afterwards, the zero-time back-extrapolation is performed by finding the instant corresponding to the envelope's peak (PEF time), calculating the Time-Volume curve, drawing the tangent at the PEF time and finding where the tangent crosses the abscissas, which is the initial instant.

The ending at noise level was detected using a sliding window algorithm (5\% of signal's length, $25 \%$ overlap) based on the magnitude ratio threshold of the maximum value $(2 \%)$.

\subsubsection{Signal Pre-processing}

The recordings are limited in excursion and patients need to perform the expiratory maneuver at an arms length to avoid microphone saturation. Therefore, it seems relevant to compensate the pressure lost between the lips $\left(p_{\text {lips }}\right)$ and smartphone $(p)$, using an Inverse Radiation Model. Furthermore, this model also 
atones the reverberation effect from sound reflections around a person's body. Afterwards, $p_{\text {lips }}$ was converted to airflow at the lips $\left(u_{\text {lips }}\right)$, using a Pressure to Flow Conversion Model. Both models were developed in similar fashion to (Larson et al., 2012).

\subsubsection{Envelope Generation}

The third stage employed several methods to calculate the signal envelopes, approaching different sound characteristics to obtain a comprehensively robust feature extraction. The algorithms' input consisted of both the segmented audio and the two resulting signals from the pre-processing stage, as all of them can be considering roughly proportional to air flow.

Generic Envelope Extraction. To obtain an envelope based on a time domain approach two methods were used: the Hilbert Transform and Shannon curves (Liang et al., 1997). The first approach consists of calculating the signal's harmonic conjugate with the Hilbert Transform and to add it back to the signal, resulting on an envelope. The second approach involves calculating the Shannon Entropy and Energy envelopes of the signal. They act as non-linear transformations focusing either on the higher (Energy) and lower (Entropy) intensities of the signal. Both approaches output highly noisy curves that need subsequent smoothing.

Linear Predictive Coding. The audio input is segmented in windows of $31.25 \mathrm{~ms}$, with $50 \%$ overlap. The white noise variance, or power, is obtained from the LPC model outputs. While the LPC filters can approximate the vocal tract (Wakita, 1973), the succession of power values should be proportional to the exhalation power at the respective time and constitute a sampled envelope of the signal. The implementation included models of degrees 2, 4, 8, 16 and 32, which represents increasing vocal complexity.

Mean of Resonances. Similarly to LPC, the signal was buffered into $31.25 \mathrm{~ms}$ frames, with $50 \%$ overlap. Each frame underwent a 256-point FFT operation using a hamming window, producing a spectrogram. All spectrogram values lower then $20 \%$ the respective frames' maximum were considered noise and were consequently discarded. Resonances over $250 \mathrm{~ms}$, within the respective frequencies' 2 bin neighborhood were kept, preserving only relatively large and long frequencies, and taking into account the natural occurring frequency shift. The envelope was obtained by averaging the frames' saved resonances.

\subsubsection{Envelope Post-processing}

The several envelopes obtained were processed using different settings in order to find the best combination for the application. The envelopes were smoothed by either a regular low pass filter (LPF) or a moving average (MA) and, in parallel, were also approximated by a 4th order polynomial. To obtain the same sampling rate as the buffered methods, the Hilbert Transform and Shannon envelopes' results were downsampled accordingly. The non-approximated envelopes were further processed using a Savitzky-Golay filter (SG) with order 3 and size 11 (Savitzky and Golay, 1964), as depicted on Figure 1.

\subsection{Parameter Extraction}

For each recording, the spirometry parameters were calculated from each of the final envelopes. The measurements extracted were PEF, FVC, $\mathrm{FEV}_{1}$, $\mathrm{FEV}_{1} / \mathrm{FVC}, \mathrm{FEF}_{25 \%-75 \%}, \mathrm{FEF}_{25 \%}, \mathrm{FEF}_{50 \%}, \mathrm{FEF}_{75 \%}$ and a custom parameter proposed in (van Stein, 2013). The envelopes are viewed as Flow-Time curves, typical of spirometer reports.

PEF is defined as the Peak Expiratory Flow or the global maximum of the audio envelope. By integrating the envelope with respect to time the Volume-Time curve can be obtained. FVC is defined as the total volume expired of a FEM. FEV 1 is the total volume expired during the first second. $\mathrm{FEF}_{25 \%-75 \%}$ corresponds to $1 / 2 \mathrm{FVC} /\left(t_{75 \%}-t_{25 \%}\right)$, in which $t_{x} \%$ is the time at which the volume corresponds to $x \%$ of the FVC. $\mathrm{FEF}_{x} \%$ is the instantaneous flow value at $\mathrm{x} \%$ of the total volume. Due to the highly noisy nature of the recordings, these last measurements were calculated as the average flow during an interval of $5 \%$ the total sound's duration, around the corresponding time instant.

\subsection{Machine Learning}

The system's machine learning pipeline can be divided into two stages: the parameter regression and the classification. The first uses the parameters extracted from the curves to obtain an estimation of the respective clinical values as given by spirometers. The second devises models that can discern between the possible illness states, initially addressing the distinction of normal from abnormal lung function and then, normal from 3 types of pathologies.

\subsubsection{Regression Stage}

Every recording produces several envelopes and each one is used to extract clinical measurements. This in- 


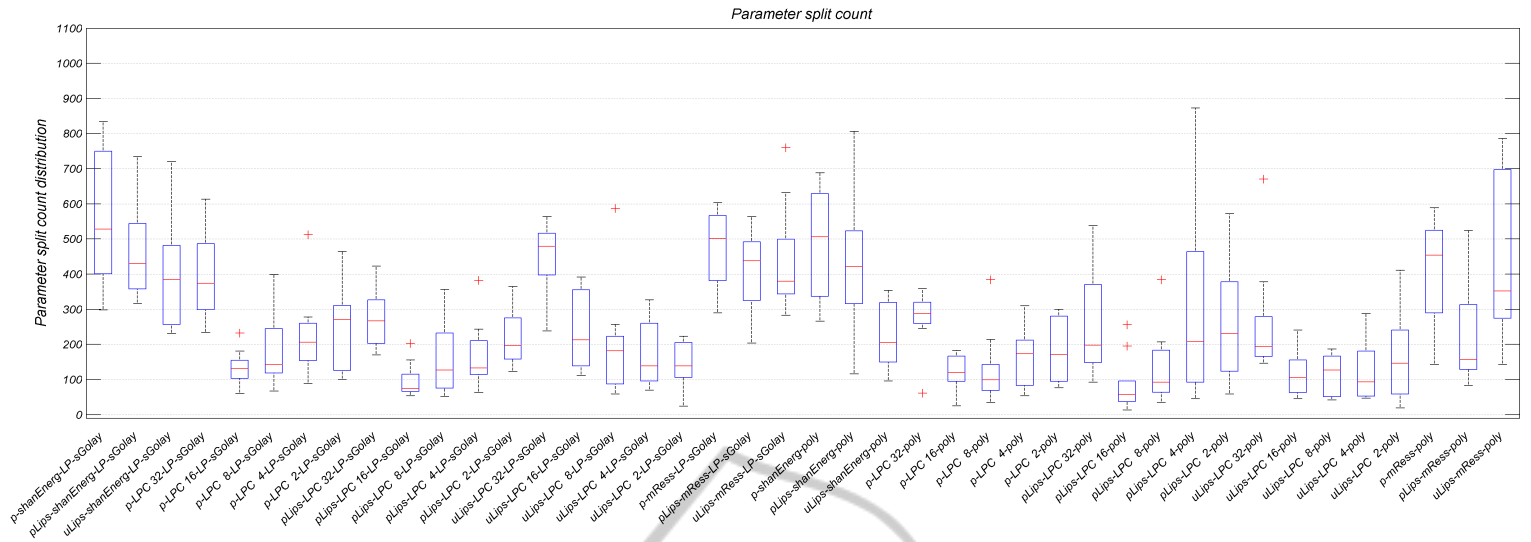

Figure 2: Split count distribution across the 9 parameters. Represents and enables to compare the gross importance of each envelope generating process.

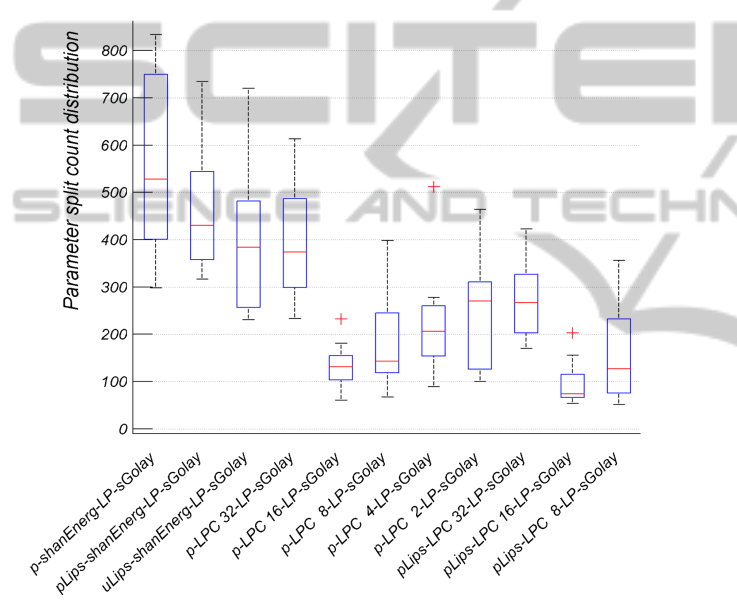

Figure 3: Enlargement of the left portion of the split count distribution graphic (refer to Figure 2).

formation is used to produce a relatively robust estimation of the respective spirometer measurement. For instance, each set of PEF measurements computed from any recording is used as a batch input for the trained regression model to obtain an estimated PEF value and the process is repeated for the other types of clinical measurements. The corresponding spirometer measurements acted as ground truth or regression targets. For this task Regression Tree Bagging (Breiman, 1996) and Random Forests (RF) (Breiman, 2001) were used in 180 tree ensembles. Also, RF employed a selection size for the random feature subset of $n / 3$ out of the total $n$ feature set.

\subsubsection{Classification Stage}

On this stage, the regressed parameters were the input of the learning models. Several different classification models were tested, namely: Decision Trees (Buntine, 1992), either as one tree, Tree Bagging, Ran- dom Forest ( $\sqrt{n}$ subset) and AdaBoost (Freund and Schapire, 1996), Support Vector Machines (SVM) (Vapnik, 1999) and Naïve Bayes (Russel and Norvig, 2002). Although the tree ensemble methods used 70 trees, only 10 trees were grown for AdaBoost to avoid overfitting. With the exception of SVM which was implemented using linear kernel models of LIBSVM, all the machine learning methods used were the default implementations available in Matlab.

\section{EXPERIMENTAL APPROACH}

\subsection{Regression Experiments}

The algorithms used on the experiments were based on a backward selection approach. Initially, all the signal processing methods were used and the clinical parameters' sets were obtained by successively removing some methods out of the initial set. For each pipeline tested, a 5-fold cross validation data set was made to verify the models' expected accuracy and to obtain the average regression error and standard deviation.

The following method comparison experiments were conducted:

1. Influence of $p_{\text {lips }}$,

2. Filtering (LPF vs. Moving Average),

3. Hilbert Transform vs. Shannon envelopes,

4. Shannon Entropy vs. Energy,

5. LPC options,

6. LPF vs. SG,

7. Influence of Polynomial fitting. 
Table 1: Comparison of regression error average and standard deviation for every parameter, and for the first (using all methods) and final signal processing pipeline.

\begin{tabular}{|c|c|c|c|c|c|c|c|c|c|c|c|}
\hline \multirow{2}{*}{$\begin{array}{l}\text { Data } \\
\text { type }\end{array}$} & \multirow[b]{2}{*}{ Exp. } & \multirow{2}{*}{ Models } & \multicolumn{9}{|c|}{ Regressed Parameters } \\
\hline & & & PEF & FVC & $\mathbf{F E V}_{1}$ & Tiff & MMEF & Cust & FEF25 & FEF50 & FEF75 \\
\hline \multirow{4}{*}{$\underset{\%}{\text { Avg. }}$} & First/ & Bag & 19.95 & 30.58 & 22.20 & 10.61 & 43.19 & 24.01 & 64.21 & 42.07 & 33.92 \\
\hline & & $\mathrm{RF}$ & 19.80 & 30.12 & 22.73 & 9.92 & 45.41 & 25.04 & 65.79 & 40.01 & 34.21 \\
\hline & & Bag & 20.22 & 30.08 & 24.58 & 10.06 & 42.74 & 23.87 & 75.56 & 42.64 & 34.09 \\
\hline & $\mathrm{F}$ & $R F$ & 20.80 & 30.38 & 23.92 & 9.56 & 43.04 & 24.66 & 67.67 & 41 & 33.06 \\
\hline \multirow{4}{*}{$\begin{array}{c}\text { Std. } \\
\%\end{array}$} & First/ & Bag & 20.64 & 27.09 & 19.85 & 8.87 & 54.65 & 27.03 & 91.28 & 48.19 & 40.65 \\
\hline & A & $\mathrm{RF}$ & 19.96 & 28.27 & 20.04 & 9.11 & 58.81 & 28.42 & 87.87 & 43.69 & 46.78 \\
\hline & & Bag & 19.33 & 28.50 & 21.14 & 8.37 & 56.42 & 25.86 & 112.39 & 50.48 & 45.75 \\
\hline & Гinal & $\mathrm{RF}$ & 21.15 & 29.18 & 21.23 & 8.88 & 54.00 & 27.17 & 90.48 & 49.37 & 41.16 \\
\hline
\end{tabular}

Two types of measurements were used to assess the method sets, the simple comparison of the regression average error, the process' tree split count and respective distribution across the parameters. Figures 2 and 3 show the split count distribution of regression Exp.5, using 5-fold bagging ensembles of 180 trees per parameter. Each box includes the 9 clinical parameters' split count for each envelope process. For instance, the first box, which corresponds to the envelope process $p \rightarrow$ Shannon Energy $\rightarrow$ LPF $\rightarrow$ SG, presents a higher split count across all parameters than the tenth box, which corresponds to the envelope process $p \rightarrow p_{\text {lips }} \rightarrow$ LPC $16 \rightarrow$ LPF $\rightarrow$ SG. This suggests that the second option is the less relevant of the two, concerning the regression models' learning process.

\subsection{Classification Experiments}

The classification process was first devised as a Normal against Abnormal classification problem, referred to as two label experiments (TLE). Then, multiple label experiments (MLE) were conducted, where the models tried to distinguish between Normal lung function and Obstruction, Restriction and Mixed pathologies. For both problems, the experiments varied on the feature space used. The experiments used:

1. Set $\mathrm{A}^{1}$ and Set $\mathrm{B}^{2}$,

2. Set A,

3. Set A, Set B and height,

4. Set A, Set B, height and age,

5. Set A, height and age.

\footnotetext{
${ }^{1}$ Set A: PEF, FEV 1 , FVC, FEV $1 /$ FVC

${ }^{2}$ Set B: $\mathrm{FEF}_{25 \%-75 \%}$, Custom, $\mathrm{FEF}_{25 \%}, \mathrm{FEF}_{50 \%}$ and $\mathrm{FEF}_{75 \%}$
}

\section{RESULTS AND DISCUSSION}

\subsection{Regression Experiments}

Concerning the regression results, Exp. 1 did not attribute significant importance to the Inverse Radiation Model and respective gain on the Pressure to Flow Conversion Model, since $p_{\text {lips }}$ did not influence the results. However, the split count results revealed that both pre-processing models' and unprocessed audio signals contributed in similar amount to the regression trees' growth. Therefore, all pre-processing models were kept.

On Exp. 2, both methods presented the same results and Moving Average was discarded since it employed a similar concept to SG. On Exp. 3, the Hilbert Transform presented the approximate same importance as the Shannon envelopes and Hilbert Transform was removed for its computational complexity. On Exp. 4, Shannon Entropy and Energy, had similar results, and Shannon Entropy was removed since it was the one with the lowest and less stable tree bagging split count.

On Exp. 5, the same split count pointed that the middle complexity LPC envelopes were less relevant and they were removed since the regression results did not change upon removal. Exp. 6 enabled to remove SG altogether due to its lower influence on the tree split count. On Exp 7 the choice of maintaining LPF over Polynomial approximation was made due to the LPF's lower computational complexity and more visual information.

Throughout the experiments, regressing the parameters using Bagging and Random Forest always presented very similar results. Therefore, the regressed parameters used on the classification experiments were obtained using Random Forest in order to reduce overfitting and shorten the regression time of the final system. Table 1 presents the regression results for the first and final experiments. Bag refers 
to Tree Bagging and $R F$ to Random Forest models. Tiff is the modified Tiffeneau index, $\mathrm{FEV}_{1} / \mathrm{FVC}$, and

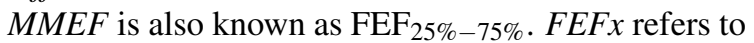
$\mathrm{FEF}_{x \%}$.

\subsection{Classification Experiments}

On the classification experiments both LPF and Polynomial Approximation parameter sets were tested on the classifiers from Exp. 1 of TLE and Exp. 5 of MLE. On the first case, the polynomial fitting presented clearly superior results than LPF, with, at least, $5 \%$ less misclassification error rate. On the second case, the results are closer between the options, differing around $2 \%$, with higher variability. All other classification experiments were conducted using the final signal processing architecture, shown on Figure 4, which disregarded LPF for Polynomial Approximation.

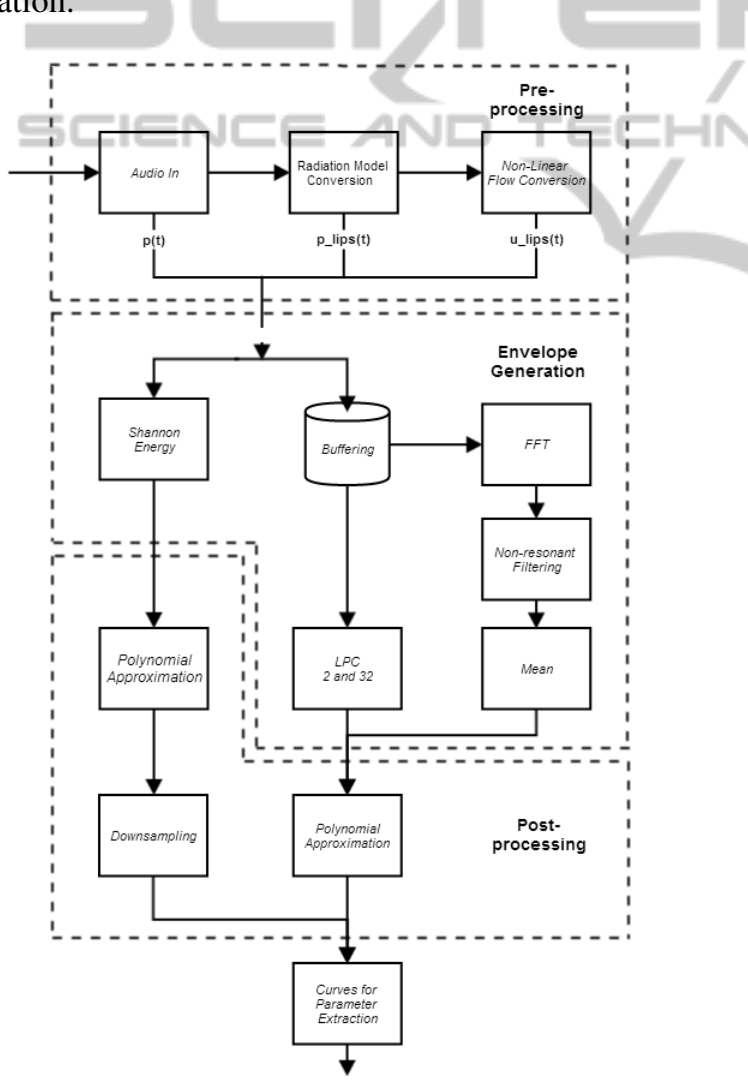

Figure 4: Final Signal Processing Architecture.

Generally and in Exp. 5 of TLE and MLE, the tree ensemble methods and Linear SVM presented the best results. For simplicity of implementation and speed of training and testing, Random Forest was chosen for the final prototype. Tables 2 and 3 present the classification results for both problems, using the final prototype. Err. refers to error rate, Prec. to Precision and Rec. to Recall. The single decision tree classifier was included for reference.

For both problems, Exp. 2 through 5 gradually improved the results across the learning methods. This indicates that the anthropometric parameters are of the utmost value when dealing with spirometric parameters. Lung volumes and flows are only relevant when considering the patient's physical characteristics.

Table 2: Two label classification problem (TLP) results (\%) for the best performing models and single tree classifier.

\begin{tabular}{|l|ccc|ccc|}
\hline \multirow{2}{*}{ Method } & \multicolumn{3}{|c|}{ TLP - Exp. 1 } & \multicolumn{3}{c|}{ TLP - Exp. 5 } \\
\cline { 2 - 7 } & Err. & Prec. & Rec. & Err. & Prec. & Rec. \\
\hline $\begin{array}{l}\text { Single } \\
\text { Tree }\end{array}$ & 29.7 & 78.1 & 80.2 & 18.8 & 86.1 & 87.3 \\
\hline $\begin{array}{l}\text { Tree } \\
\text { Bagging }\end{array}$ & 24.7 & 81.1 & 84.5 & 15.8 & 86.6 & 91.5 \\
\hline $\begin{array}{l}\text { Random } \\
\text { Forest }\end{array}$ & 29.7 & 79.7 & 77.4 & 7.9 & 90.9 & 98.5 \\
\hline Adaboost & 32.6 & 79.6 & 71.8 & 9.9 & 92.9 & 92.9 \\
\hline SVM & 29.7 & 78.1 & 80.2 & 8.9 & 90.7 & 97.1 \\
\hline
\end{tabular}

Table 3: Multiple label classification problem (MLP) results for the best performing models and single tree classifier.

\begin{tabular}{|l|c|c|}
\hline \multirow{2}{*}{ Method } & \multicolumn{2}{|c|}{ Error Rate \% } \\
\cline { 2 - 3 } & $\begin{array}{c}\text { MLP } \\
\text { Exp. 1 }\end{array}$ & $\begin{array}{c}\text { MLP } \\
\text { Exp. 5 }\end{array}$ \\
\cline { 2 - 3 } $\begin{array}{l}\text { Single } \\
\text { Tree }\end{array}$ & 44.5 & 27.7 \\
\hline $\begin{array}{l}\text { Tree } \\
\text { Bagging }\end{array}$ & 27.7 & 19.8 \\
\hline $\begin{array}{l}\text { Random } \\
\text { Forest }\end{array}$ & 27.7 & 17.8 \\
\hline Adaboost & 27.7 & 19.8 \\
\hline SVM & 33.6 & 14.8 \\
\hline
\end{tabular}

\subsection{Analysis of the Regression Results using the Final Architecture}

Once the signal processing architecture is defined it is important to evaluate whether the lung function parameters are under-estimated or over-estimated. Figure 5 shows Bland-Altman plots (Bland and Altman, 1986) of the 4 most popular clinical parameters, distinguishing between the spirometer target and the regressed values versus the mean between the target and regressed values. Also, the average error (dashdot) and the $\pm 2 \sigma$ (dash) indicative lines are shown.

The error average lines are close to zero, which speaks to the validity of the regression models. However, the relatively high standard deviation $( \pm 1.6 \mathrm{~L} / \mathrm{s}$, $1 \mathrm{~L}, 10 \%)$, indicates that they are still not very accurate. Generally, these plots show that the regression models tend to over-estimate the spirometers mea- 

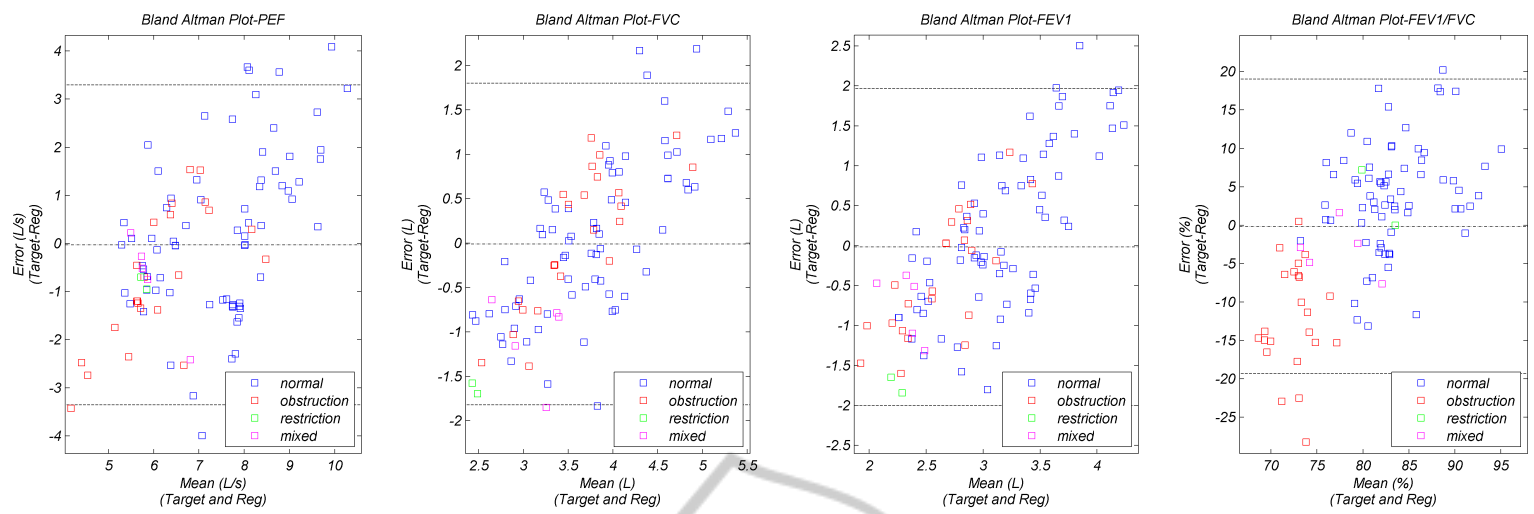

Figure 5: Bland Altman plots of the errors between the spirometer target and the regressed values versus the mean between the target and regressed values. The mean (dashdot) and $\pm 2 \sigma$ (dash) are also shown.

surements of the lower volume or flow samples.

There are a few outliers which present around or over $100 \%$ error, particularly on the PEF results. This could be explained considering that the PEF measurement is, essentially, the global maximum of an envelope, which is highly dependent of both audio and envelope filtering methods. On the other hand, the PEF is a cooperation dependent measurement and may induce error on the learning models. Concerning the volume dependent measurements, $\mathrm{FEV}_{1}$ and FVC, the outliers comprise the lung restriction class samples, that are characterized by lower target volumes, which are clearly over-estimated.

\section{LIMITATIONS AND FUTURE WORK}

\subsection{Limitations}

This project presented some issues concerning data collection that, once overcome, should enhance the learning models' performance and, consequently, the results.

A great portion of the recordings was gathered on a relatively fast paced clinical study where patients had to perform several respiration maneuvers before recording to the smartphone. This could have reduced the patient's cooperation level due to fatigue. On the other hand, the forced expiration maneuver itself is difficult to perform, specially when concerning these recordings where no mouthpiece was used. These factors also contributed to the reduced yield of properly executed recordings. The small dataset with little intra-patient samples is most likely the cause of the regression errors of over $20 \%$.

Additionally, since the spirometer and smartphone maneuvers were made separately there is no completely reliable ground truth.

\subsection{Future Work}
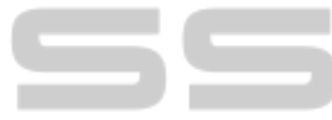

Further study of this technology is needed and some key features and experiments are proposed. First, some relevant combinations of methods were left untested and, provided additional data is gathered, would be interesting to evaluate. Second, it would be relevant to devise an algorithm to automatically detect poorly executed FEMs. Finally, an application based on the proposed architecture should be implemented.

\section{CONCLUSION}

The regression experiments enabled to reduce the 54 to 12 final envelopes, per recording, without a significant change on the results. The regression mean error for the less error prone parameters was $21 \%, 30 \%$, $24 \%$ and $9.6 \%$, for $\mathrm{PEF}, \mathrm{FEV}_{1}, \mathrm{FVC}$, and $\mathrm{FEV}_{1} / \mathrm{FVC}$, respectively. The classification models obtained an error rate of $8 \%$ and $18 \%$, for the TLE and MLE, respectively. Also, the TLE model presented a precision of $90.91 \%$ and recall of $98.59 \%$. In conclusion, our work demonstrated that smartphone spirometry and automatic lung function triage is possible and the results encourage further development of the systems.

\section{REFERENCES}

Bland, J. M. and Altman, D. G. (1986). Statistical methods for assessing agreement between two methods of clinical measurement. Lancet, 1(8476):307-10.

Breiman, L. (1996). Bagging predictors. Machine Learning, 24(2):123-140. 
Breiman, L. (2001). Random forests. Machine learning, 45(1):5--32.

Buntine, W. (1992). Learning classification trees. Statistics and Computing, 2(2):63-73.

Freund, Y. and Schapire, R. E. (1996). Experiments with a new boosting algorithm. In Saitta, L., editor, ICML, pages 148-156, Bari, Italy. Morgan Kaufmann.

Larson, E. C., Goel, M., Boriello, G., Heltshe, S., Rosenfeld, M., and Patel, S. N. (2012). SpiroSmart: Using a Microphone to Measure Lung Function on a Mobile Phone. In 14th ACM International Conference on Ubiquitous Computing, page 10, Pittsburgh, Pennsylvania, USA.

Liang, H., Lukkarinen, S., and Hartimo, I. (1997). Heart sound segmentation algorithm based on heart sound envelogram. In Computers in Cardiology 1997, volume 24, pages 105-108. IEEE.

Miller, M. R., Hankinson, J., Brusasco, V., Burgos, F., Casaburi, R., Coates, A., Crapo, R., Enright, P., van der Grinten, C. P. M., Gustafsson, P., Jensen, R., Johnson, D. C., MacIntyre, N., McKay, R., Navajas, D., Pedersen, O. F., Pellegrino, R., Viegi, G., and Wanger, J. (2005). Standardisation of spirometry. The European respiratory journal, 26(2):319-38.

Pierce, R. (2005). Spirometry: an essential clinical measurement. Australian family physician, 34(7):535-9.

Russel, S. and Norvig, P. (2002). Artificial Intelligence: A Modern Approach. Prentice Hall, 2nd edition.

Savitzky, A. and Golay, M. J. E. (1964). Smoothing and Differentiation of Data by Simplified Least Squares Procedures. Analytical Chemistry, 36(8):1627-1639.

van Stein, B. (2013). A Mobile Smart Care platform Home spirometry by using the smartphone microphone. Master's thesis, Leiden University, Leiden, The Netherlands.

Vapnik, V. N. (1999). An overview of statistical learning theory. IEEE transactions on neural networks / a publication of the IEEE Neural Networks Council, 10(5):988-99.

Wakita, H. (1973). Direct estimation of the vocal tract shape by inverse filtering of acoustic speech waveforms. Audio and Electroacoustics, IEEE Transactions on, 21(5):417-427.

World Health Organization (2013a). Asthma: $\quad$ Fact sheet N307. http://www.who.int/mediacentre/factsheets/fs307/en/ . Accessed: 26-06-2014.

World Health Organization (2013b). Chronic obstructive pulmonary disease (COPD): Fact sheet N315. http://www.who.int/mediacentre/factsheets/fs315/en/ . Accessed: 26-06-2014.

Xu, W., Huang, M.-c., Liu, J. J., Ren, F., Shen, X., Liu, X., and Sarrafzadeh, M. (2013). mCOPD. In Proceedings of the 6th International Conference on Pervasive Technologies Related to Assistive Environments - PETRA '13, PETRA '13, pages 1-8, New York, New York, USA. ACM Press. 